\title{
Ultrafast imaging of light scattering dynamics using second-generation compressed ultrafast photography
}

Jinyang Liang, Cheng Ma, Liren Zhu, Yujia Chen, Liang Gao, et al.

Jinyang Liang, Cheng Ma, Liren Zhu, Yujia Chen, Liang Gao, Lihong V. Wang, "Ultrafast imaging of light scattering dynamics using second-generation compressed ultrafast photography," Proc. SPIE 10076, High-Speed Biomedical Imaging and Spectroscopy: Toward Big Data Instrumentation and Management II, 1007612 (22 February 2017); doi: 10.1117/12.2250198

SPIE. Event: SPIE BiOS, 2017, San Francisco, California, United States 


\title{
Ultrafast imaging of light scattering dynamics using second-generation compressed ultrafast photography \\ Jinyang Liang, Cheng Ma, Liren Zhu, Yujia Chen, Liang Gao, and Lihong V. Wang* \\ Optical Imaging Laboratory, Department of Biomedical Engineering, Washington University in St. Louis, Campus Box 1097, One Brookings Drive, St. Louis, Missouri 63130, USA
}

\begin{abstract}
We present single-shot real-time video recording of light scattering dynamics by second-generation compressed ultrafast photography (G2-CUP). Using G2-CUP at 100 billion frames per second, in a single camera exposure, we experimentally captured the evolution of the light intensity distribution in an engineered thin scattering plate assembly. G2-CUP, which implements a new reconstruction paradigm and a more efficient hardware design than its predecessors, markedly improves the reconstructed image quality. The ultrafast imaging reveals the instantaneous light scattering pattern as a photonic Mach cone. We envision that our technology will find a diverse range of applications in biomedical imaging, materials science, and physics.
\end{abstract}

Keywords: ultrafast imaging, compressed sensing, streak camera, image reconstruction techniques

\section{INTRODUCTION}

In recent years, light scattering has been extensively exploited in many biomedical optical imaging modalities [1-4]. For instance, techniques for spatiotemporally inverting light scattering have allowed focusing light into deep tissue for highresolution imaging and control [5,6]. Ultrafast imaging of non-repetitive dynamic light scattering is becoming increasingly important for next-generation biomedical optical instrumentation. In addition, analysis of temporal fluctuations in scattered light signals reveals many optical properties of biological tissues $[1,7]$. This characterization has enabled diverse applications, such as assessments of food and pharmaceutical products [8] and studies of protein aggregation diseases $[9,10]$.

Light scattering dynamics have been extensively investigated from both theoretical and experimental perspectives. Among many simulation paradigms [11-13], the Monte Carlo method offers a rigorous and flexible approach [14] and often is regarded as the gold standard for modeling light transport in a scattering medium [15]. A Monte Carlo simulation is equivalent to modeling photon transport analytically by solving the radiative transfer equation [16]. As a statistical approach, a typical Monte Carlo simulation provides an ensemble-averaged result of light propagation (i.e., it ignores coherent effects [17]) and requires launching a large number of photons to ensure the desired accuracy [18]. The Monte Carlo method can simulate light propagation sequences with a short (e.g., sub-nanosecond) time interval. This time-resolved Monte Carlo simulation has been used widely to model time-dependent light distribution, dynamic optical properties, and frequency domain light transportation in scattering media [19].

On the other hand, experimental visualization of light propagation in a scattering medium has been a long-standing challenge [20]. Freezing light's motion in a tabletop scene requires a picosecond-level exposure time (a billion frames per second) [21]. Despite continuous improvements in state-of-the-art electronic sensors, current CMOS and CCD technologies cannot reach this speed [22], because they are fundamentally impeded by their on-chip storage capacity and electronic readout speeds [23]. The introduction of the streak camera broke this speed limit [24]. With superior temporal resolution, the streak camera opens up new opportunities for experimental capture of light propagation in scattering media [25-29]. However, the conventional operation of the streak camera sacrifices the imaging dimension — the narrow entrance slit (10-50 $\mu \mathrm{m}$ wide) confines the imaging field of view to a line. To achieve two-dimensional ultrafast

*lhwang@wustl.edu; phone 314 935-6152; fax 314 935-7448

High-Speed Biomedical Imaging and Spectroscopy: Toward Big Data Instrumentation and Management II edited by Kevin K. Tsia, Keisuke Goda, Proc. of SPIE Vol. 10076, 1007612 · C 2017 SPIE CCC code: $1605-7422 / 17 / \$ 18 \cdot$ doi: $10.1117 / 12.2250198$ 
imaging, it is necessary to scan the orthogonal spatial dimension and synthesize the movie from a large number of measurements. Despite its capability of capturing photons in motion, this technique requires that the scattering events be precisely repeatable, which is inherently challenging for dynamic scattering media such as soft biological tissues and flowing blood.

Overcoming these limitations, here we present single-shot real-time video recording of spatiotemporal light patterns in a scattering medium. We developed a second-generation compressed ultrafast photography (G2-CUP) system, whose more efficient hardware design and reconstruction paradigm surpass the performance of previous CUP systems [30-32]. At 100 billion frames per second, G2-CUP macroscopically imaged the evolution of the light intensity distribution of a picosecond laser pulse propagating in a thin scattering plate assembly that contains two materials with different refractive indices. The instantaneous scattering pattern is depicted as a photonic Mach cone [33, 34].

\section{DEVELOPMENT OF G2-CUP}

\subsection{Principle of Operation}

As a computational imaging approach, G2-CUP involves physical data acquisition and computational image reconstruction. In data acquisition, the scene is imaged in three views. One view is directly recorded by an external CCD camera as the time-unsheared view, and the measured optical energy distribution is denoted $E^{(0)}$. To obtain two timesheared views, the image is first spatially encoded by a pair of complementary pseudo-random binary patterns, then temporally sheared along the vertical spatial axis using a streak camera, and finally imaged to the internal CCD camera of the streak camera as two optical energy distributions, $E^{(1)}$ and $E^{(2)}$. Mathematically, the three views can be related to the intensity distribution of the dynamic scene $I(x, y, t)$ as follows:

$$
E=\boldsymbol{O} I
$$

where $E=\left[E^{(0)}, \alpha E^{(1)}, \alpha E^{(2)}\right]^{T}$ and $\boldsymbol{O}=\left[\boldsymbol{T} \boldsymbol{F}_{\mathbf{0}}, \alpha \boldsymbol{T} \boldsymbol{S} \boldsymbol{D}_{\mathbf{1}} \boldsymbol{F}_{\mathbf{1}} \boldsymbol{C}_{\mathbf{1}}, \alpha \boldsymbol{T} \boldsymbol{S} \boldsymbol{D}_{\mathbf{2}} \boldsymbol{F}_{\mathbf{2}} \boldsymbol{C}_{\mathbf{2}}\right]^{T}$. The scalar factor $\alpha$ is related to the energy calibration of the streak camera against the external CCD camera. The linear operator $\boldsymbol{T}$ represents spatiotemporal integration, $\boldsymbol{F}_{\boldsymbol{j}}(j=0,1,2)$ represents spatial low-pass filtering, $\boldsymbol{S}$ represents temporal shearing, $\boldsymbol{D}_{\boldsymbol{i}}(i=1,2)$ represents image distortion due primarily to the encoding arm, and $\boldsymbol{C}_{\boldsymbol{i}}(i=1,2)$ represents complementary spatial encoding with $C_{1}+C_{2}=1$.

With prior knowledge of $\boldsymbol{O}, I(x, y, t)$ can be estimated from measurements from the three views, $E$, by solving the inverse problem of Eq. 1. Because of the sparsity in the input scene, the image reconstruction can be realized by solving the following optimization problem:

$$
\hat{I}=\arg \min _{I}\left[\frac{1}{2}\|E-\boldsymbol{O} I\|^{2}+\beta \Phi(I)\right]
$$

where the first term $\frac{1}{2}\|E-\boldsymbol{O} I\|^{2}$ represents the measurement fidelity, and the regularization term $\Phi(I)$ encourages sparsity. The regularization parameter $\beta$ adjusts the weight ratio between fidelity and sparsity. For G2-CUP image reconstruction, a compressed-sensing reconstruction algorithm developed from two-step iterative shrinkage/thresholding (TwIST) algorithm [35] was employed.

\subsection{System details}

In the G2-CUP system, the dynamic scene is first imaged by a camera lens (Fujinon CF75HA-1) with a focal length of $75 \mathrm{~mm}$. Following the intermediate imaging plane (Fig. 1), a beam splitter (Thorlabs BS013) reflects half of the light to an external CCD camera (Point Grey GS3-U3-28S4M-C). The other half of the light passes through the beam splitter and is imaged to a digital micromirror device (DMD, Texas Instruments DLP LightCrafter 3000) through a 4f system consisting of a tube lens (Thorlabs AC508-100-A) and a stereoscope objective (Olympus MV PLAPO 2XC; NA = 0.50). The spatially encoded images are projected to the entrance slit of a streak camera (Hamamatsu C7700) through two more $4 f$ systems containing the same stereoscope objective, tube lenses (Thorlabs AC254-75-A), planar mirrors, and a right- 
angle prism mirror. The shearing velocity of the streak camera is set to $v=1.32 \mathrm{~mm} / \mathrm{ns}$. The spatially encoded, temporally sheared images are acquired by an internal CCD camera (Hamamatsu, ORCA-R2) with a sensor size of $672 \times 512$ binned pixels $(2 \times 2$ binning, binned pixel size $d=12.9 \mu \mathrm{m})$. The reconstructed frame rate, $\mathrm{r}$, is determined by $r=v / d$ to be 100 billion frames per second.

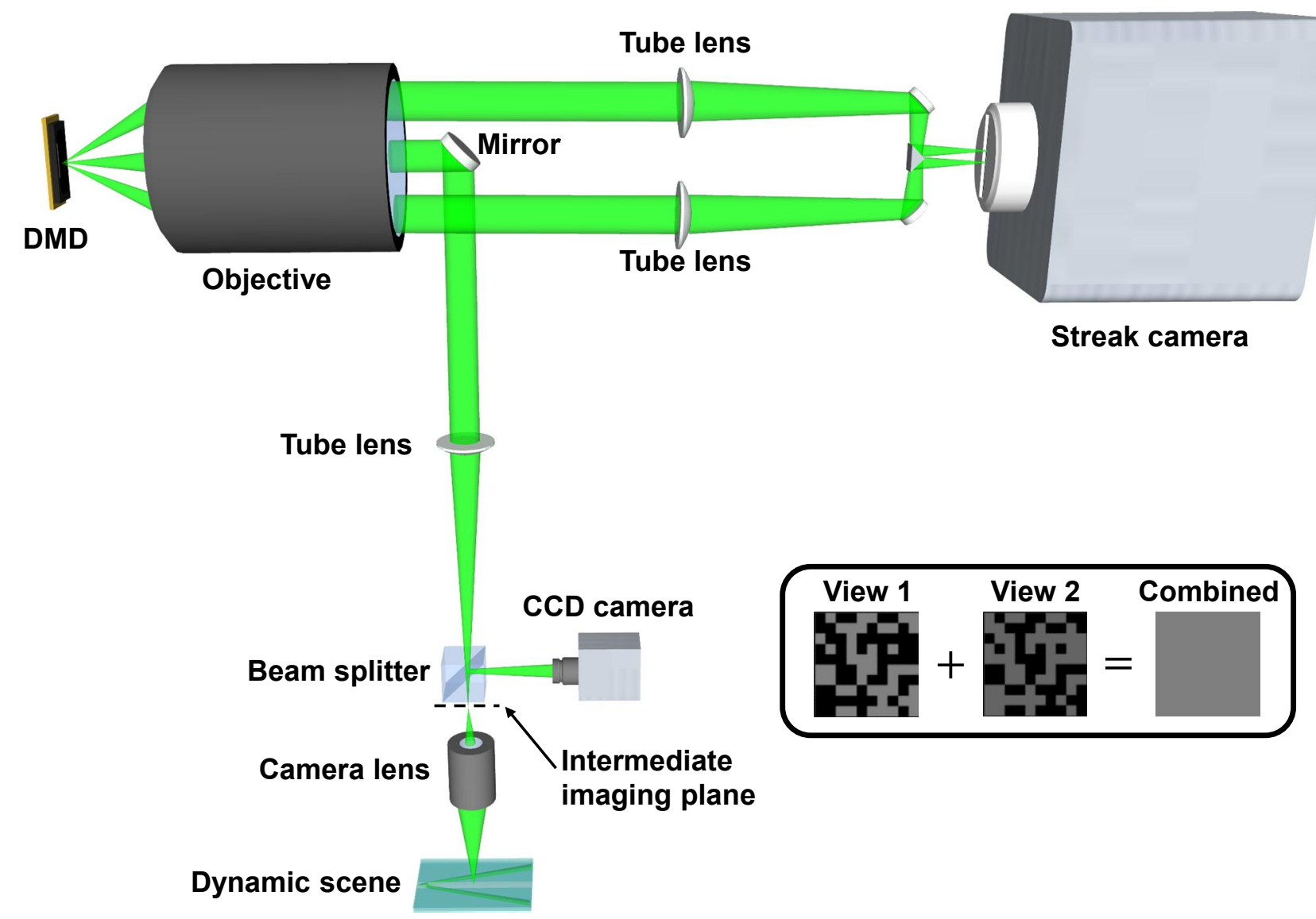

Figure 1. Schematic of second-generation compressed ultrafast photography. DMD, digital micromirror device. Inset: Illustration of complementary DMD masks for loss-less encoding of time-unsheared views.

\subsection{Comparison to previous CUP systems}

In previously reported CUP systems [30-32], only the "ON" pixels of the DMD were used in the spatial encoding operation. As a result, information that landed on the "OFF" pixels of the DMD was lost, compromising reconstruction quality. In addition, the time-integrated CCD image was simply overlaid with the reconstructed datacube as a postprocessing step [31], without adding new information to assist image reconstruction. In contrast, G2-CUP harvests light reflected from both the "ON" and "OFF" pixels of the DMD, forming two complementary time-sheared views. This design prevents loss of information from spatial encoding, which is advantageous for compressed sensing based reconstruction. In addition, the time-unsheared view recorded by the external CCD camera enriches the observation by adding another perspective, which is used with the two time-sheared views in the new reconstruction paradigm to yield much improved image quality. Thus, the dual complementary masking, the triple-view recording of the scene, and the three-view joint reconstruction are three major improvements of G2-CUP over the previous CUP systems. 


\section{IMAGING PHOTONIC MACH CONES}

Of particular interest is the long-sought-after transient phenomenon-photonic Mach cones [33, 34]. Although their propagation has been previously observed using pump-probe methods [36, 37], a single-shot, real-time observation of travelling photonic Mach cones has not yet been achieved. To tackle this challenge, we generated a photonic Mach cone by scattering a picosecond laser pulse traveling superluminally relative to the surrounding medium. Specifically, we built a thin scattering plate assembly containing a central source tunnel sandwiched between two display panels (Fig. 1A). The scattering plate assembly was built in three steps. First, we used standard microscope cover slides to make a display panel with cavity dimensions of $75 \mathrm{~mm} \times 50 \mathrm{~mm} \times 4 \mathrm{~mm}$. Then, to scatter light, we mixed $50 \mathrm{mg}$ of aluminum oxide $\left(\mathrm{Al}_{2} \mathrm{O}_{3}\right)$ powder (Sigma-Aldrich, 265497) into $20 \mathrm{~mL}$ of liquid silicone rubber (Wacker Silicone, Elastosil RT 601A, refractive index $n_{d}=1.4$ ). After complete mixing, we added rubber solidifier (Wacker Silicone, Elastosil RT 601B) and poured the mixture into the two display panel cavities. In the top panel, the $\mathrm{Al}_{2} \mathrm{O}_{3}$-rubber scattering material filled the entire panel cavity. At the center of the display panel assembly, a 4-mm deep space was left at the top as the source tunnel. Both panels were left to solidify for 24 hours. Finally, we used air (refractive index $n_{d}=1.0$ ) with dry ice fog in the source tunnel.

A collimated visible laser pulse (532 $\mathrm{nm}$ wavelength, $7 \mathrm{ps}$ pulse duration, $4 \mu \mathrm{J}$ pulse energy) was propagated through the source tunnel. The scatterers in the plate assembly extracted photons which the G2-CUP system used to image this dynamic scene. No averaging or gating over multiple laser pulses was required.
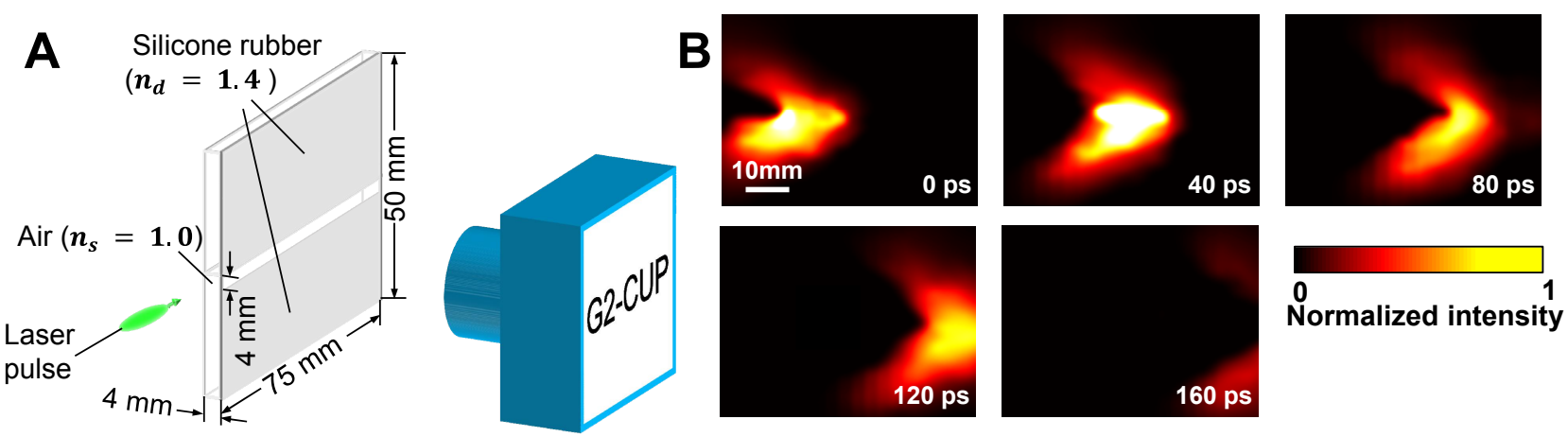

Figure 2. Imaging photonic Mach cones using G2-CUP. (A) Experimental setup. (B) Representative snapshots of scattered light intensity distribution when a laser beam propagated faster in the source tunnel $\left(n_{\mathrm{S}}=1.0\right)$ than scattered light did in the display panels $\left(n_{\mathrm{d}}=1.4\right)$. A photonic Mach cone is clearly observed.

Figure 2B shows five representative time-lapse frames of the scattered light distribution of the same dynamic event imaged by the G2-CUP system, revealing a propagating photonic Mach cone. The central $x-y$ cross section of the photonic Mach cone is displayed by the scattering plate assembly. The cone edge is seen as two light tails extending from the tip of the propagating laser pulse in the source tunnel, forming a V-shaped wedge. The semivertex angle, directly measured in these temporal frames, is $\sim 45$ degrees, which agrees with the theoretical value [38]. It is worth noting that the video-recorded light pattern shows an asymmetric spatial intensity distribution, which is probably attributable to unequal couplings of the non-uniform scattering in the source tunnel to the upper and lower display panels.

\section{SUMMARY}

We have demonstrated ultrafast video recording of light scattering dynamics using G2-CUP and, in a single camera exposure, visualized the propagation of a scattering-induced photonic Mach cone as an instantaneous light scattering pattern. Single-shot real-time video recording of light scattering dynamics is a significant contribution of the nextgeneration of imaging modalities. For example, by leveraging the time-of-flight light signal, the technology can resolve depth information without motion blurring [31]. Coupling the current system with a femtosecond streak camera [39] 
might achieve an axial resolution comparable to that of optical coherence tomography [40], allowing single-shot fullfield imaging of 3D microstructures in biological systems in vivo.

\section{ACKNOWLEDGMENT}

The authors thank Prof. James Ballard for close reading of the manuscript. This work was supported in part by National Institutes of Health grants DP1 EB016986 (NIH Director's Pioneer Award) and R01 CA186567 (NIH Director's Transformative Research Award).

\section{REFERENCES}

[1] A. Wax, and V. Backman, [Biomedical Applications of Light Scattering] McGraw Hill Professional, (2009).

[2] A. Obeid, N. Barnett, G. Dougherty, and G. Ward, "A critical review of laser Doppler flowmetry," Journal of medical engineering \& technology, 14(5), 178-181 (1990).

[3] T. Durduran, R. Choe, W. B. Baker, and A. G. Yodh, "Diffuse optics for tissue monitoring and tomography," Reports on Progress in Physics, 73(7), 076701 (2010).

[4] D. Huang, E. Swanson, C. Lin, J. Schuman, W. Stinson, W. Chang, M. Hee, T. Flotte, K. Gregory, C. Puliafito, and J. G. Fujimoto, "Optical coherence tomography," Science, 254(5035), 1178-1181 (1991).

[5] A. P. Mosk, A. Lagendijk, G. Lerosey, and M. Fink, "Controlling waves in space and time for imaging and focusing in complex media," Nat Photon, 6(5), 283-292 (2012).

[6] D. J. McCabe, A. Tajalli, D. R. Austin, P. Bondareff, I. A. Walmsley, S. Gigan, and B. Chatel, "Spatiotemporal focusing of an ultrafast pulse through a multiply scattering medium," Nat Commun, 2, 447 (2011).

[7] B. J. Berne, and R. Pecora, [Dynamic light scattering: with applications to chemistry, biology, and physics] Courier Corporation, (1976).

[8] I. Bargigia, A. Tosi, A. B. Shehata, A. Della Frera, A. Farina, A. Bassi, P. Taroni, A. Dalla Mora, F. Zappa, R. Cubeddu, and A. Pifferi, "Time-resolved diffuse optical spectroscopy up to $1700 \mathrm{~nm}$ by means of a time-gated InGaAs/InP single-photon avalanche diode,” Applied spectroscopy, 66(8), 944-950 (2012).

[9] J. J. McManus, A. Lomakin, O. Ogun, A. Pande, M. Basan, J. Pande, and G. B. Benedek, "Altered phase diagram due to a single point mutation in human $\gamma$ D-crystallin," Proceedings of the National Academy of Sciences, 104(43), 16856-16861 (2007).

[10] J. D. Gunton, A. Shiryayev, and D. L. Pagan, [Protein condensation: kinetic pathways to crystallization and disease] Cambridge university press, (2007).

[11] L. Wang, S. L. Jacques, and L. Zheng, "MCML—Monte Carlo modeling of light transport in multi-layered tissues," Computer Methods and Programs in Biomedicine, 47(2), 131-146 (1995).

[12] R. Drezek, A. Dunn, and R. Richards-Kortum, "Light scattering from cells: finite-difference time-domain simulations and goniometric measurements," Applied Optics, 38(16), 3651-3661 (1999).

[13] M. Schweiger, S. R. Arridge, M. Hiraoka, and D. T. Delpy, "The finite element method for the propagation of light in scattering media: Boundary and source conditions," Medical Physics, 22(11), 1779-1792 (1995).

[14] S. T. Flock, M. S. Patterson, B. C. Wilson, and D. R. Wyman, "Monte Carlo modeling of light propagation in highly scattering tissues. I. Model predictions and comparison with diffusion theory," IEEE Transactions on Biomedical Engineering, 36(12), 1162-1168 (1989).

[15] C. Zhu, and Q. Liu, "Review of Monte Carlo modeling of light transport in tissues," Journal of Biomedical Optics, 18(5), 050902-050902 (2013).

[16] L. V. Wang, and H. I. Wu, [Radiative transfer equation and diffusion theory] John Wiley \& Sons, 5 (2012).

[17] X. Wang, L. V. Wang, C.-W. Sun, and C.-C. Yang, "Polarized light propagation through scattering media: time-resolved Monte Carlo simulations and experiments," Journal of Biomedical Optics, 8(4), 608-617 (2003).

[18] V. V. Tuchin, [Methods and Algorithms for the Measurement of the Optical Parameters of Tissues] SPIE press Bellingham, 7 (2015).

[19] S. L. Jacques, [Monte Carlo modeling of light transport in tissue (steady state and time of flight)] Springer, 5 (2010).

[20] M. E. Zevallos L., S. K. Gayen, M. Alrubaiee, and R. R. Alfano, "Time-gated backscattered ballistic light imaging of objects in turbid water,” Applied Physics Letters, 86(1), 011115 (2005). 
[21] G. Gariepy, N. Krstajic, R. Henderson, C. Li, R. R. Thomson, G. S. Buller, B. Heshmat, R. Raskar, J. Leach, and D. Faccio, "Single-photon sensitive light-in-fight imaging," Nature Communications, 6, (2015).

[22] Y. Kondo, K. Takubo, H. Tominaga, R. Hirose, N. Tokuoka, Y. Kawaguchi, Y. Takaie, A. Ozaki, S. Nakaya, F. Yano, and T. Daigen, "Development of "HyperVision HPV-X" high-speed video camera," Shimadzu Review, 69, 285-291 (2012).

[23] M. El-Desouki, M. Jamal Deen, Q. Fang, L. Liu, F. Tse, and D. Armstrong, "CMOS image sensors for high speed applications," Sensors, 9(1), 430-444 (2009).

[24] [Guide to Streak Cameras], Hamamatsu Corp.(2008).

[25] B. B. Das, L. Feng, and R. R. Alfano, "Time-resolved fluorescence and photon migration studies in biomedical and model random media," Reports on Progress in Physics, 60(2), 227 (1997).

[26] K. M. Yoo, and R. R. Alfano, "Time-resolved coherent and incoherent components of forward light scattering in random media," Optics Letters, 15(6), 320-322 (1990).

[27] J. Wu, L. Perelman, R. R. Dasari, and M. S. Feld, "Fluorescence tomographic imaging in turbid media using early-arriving photons and Laplace transforms," Proceedings of the National Academy of Sciences, 94(16), 8783-8788 (1997).

[28] R.-J. Kutta, T. Langenbacher, U. Kensy, and B. Dick, "Setup and performance of a streak camera apparatus for transient absorption measurements in the ns to ms range," Applied Physics B, 111(2), 203-216 (2013).

[29] G. Satat, B. Heshmat, C. Barsi, D. Raviv, O. Chen, M. G. Bawendi, and R. Raskar, "Locating and classifying fluorescent tags behind turbid layers using time-resolved inversion," Nature Communications, 6, (2015).

[30] L. Gao, J. Liang, C. Li, and L. V. Wang, "Single-shot compressed ultrafast photography at one hundred billion frames per second," Nature, 516(7529), 74-77 (2014).

[31] J. Liang, L. Gao, P. Hai, C. Li, and L. V. Wang, "Encrypted Three-dimensional Dynamic Imaging using Snapshot Time-of-flight Compressed Ultrafast Photography," Scientific Reports, 5, 15504 (2015).

[32] L. Zhu, Y. Chen, J. Liang, L. Gao, C. Ma, and L. V. Wang, "Space- and intensity-constrained reconstruction for compressed ultrafast photography," Optica, 3(7), 694-697 (2016).

[33] D. H. Auston, and M. C. Nuss, "Electrooptical generation and detection of femtosecond electrical transients," IEEE Journal of Quantum Electronics, 24(2), 184-197 (1988).

[34] P. A. Čerenkov, "Visible Radiation Produced by Electrons Moving in a Medium with Velocities Exceeding that of Light," Physical Review, 52(4), 378-379 (1937).

[35] J. M. Bioucas-Dias, and M. A. T. Figueiredo, "A New TwIST: Two-Step Iterative Shrinkage/Thresholding Algorithms for Image Restoration," IEEE Transactions on Image Processing, 16(12), 2992-3004 (2007).

[36] R. M. Koehl, S. Adachi, and K. A. Nelson, "Direct Visualization of Collective Wavepacket Dynamics," The Journal of Physical Chemistry A, 103(49), 10260-10267 (1999).

[37] Z. Wang, F. Su, and F. A. Hegmann, "Ultrafast imaging of terahertz Cherenkov waves and transition-like radiation in LiNbO3," Optics Express, 23(6), 8073-8086 (2015).

[38] J. Liang, C. Ma, L. Zhu, Y. Chen, L. Gao, and L. V. Wang, "Single-shot real-time video recording of photonic Mach cone induced by a scattered light pulse," Science Advances, 3, e1601814 (2017).

[39] [FESCA-200 Femtosecond streak camera], Hamamatsu Corp.(2015).

[40] J. G. Fujimoto, C. Pitris, S. A. Boppart, and M. E. Brezinski, "Optical coherence tomography: an emerging technology for biomedical imaging and optical biopsy," Neoplasia, 2(1), 9-25 (2000). 